\title{
Quantum versus classical protons in pure and salty ice under pressure
}

\author{
Yael Bronstein, ${ }^{1}$ Philippe Depondt, ${ }^{1}$ Livia E. Bove, ${ }^{2,3}$ Richard Gaal, ${ }^{3}$ Antonino Marco Saitta, ${ }^{2}$ and Fabio Finocchi ${ }^{1}$ \\ ${ }^{1}$ Sorbonne Universités, UPMC Université Paris 06, CNRS-UMR 7588, Institut des NanoSciences de Paris, F-75005 Paris, France \\ ${ }^{2}$ Sorbonne Universités, UPMC Université Paris 06, Muséum National d'Histoire Naturelle, IRD, CNRS-UMR 7590, Institut de Minéralogie, \\ de Physique des Matériaux et de Cosmochimie, F-75005 Paris, France \\ ${ }^{3}$ École Polytechnique Fédérale de Lausanne, Institute of Condensed Matter Physics, EPFL, CH-1015 Lausanne, Switzerland
}

(Received 9 July 2015; revised manuscript received 27 November 2015; published 8 January 2016)

\begin{abstract}
It is generally accepted that nuclear quantum effects (NQEs) trigger the transition to the nonmolecular form of ice under increasing pressure. This picture is challenged in salty ice, where Raman scattering measurements up to $130 \mathrm{GPa}$ of molecular ice VII containing $\mathrm{NaCl}$ or $\mathrm{LiCl}$ impurities show that the transition pressure to the symmetric phase ice $\mathrm{X}$ is shifted up by about $30 \mathrm{GPa}$, even at small salt concentrations. We address the question of how the inclusion of salt induces the drastic reduction of NQEs by selectively including NQEs in $a b$ initio calculations of ice in the presence of distinct ionic impurities. We quantitatively show that this is mainly a consequence of the electric field generated by the ions. We propose a simple model that is able to capture the essence of this phenomenon, generalizing this picture to other charged defects and for any concentration. This result is potentially generalizable to most "dirty" ices in which the electric field due to the doping is much more significant than local lattice distortions.
\end{abstract}

DOI: 10.1103/PhysRevB.93.024104

Ice under extreme conditions is present in many planets, both within our solar system [1] and beyond [2]. These ices are usually "dirty": Planetary real ices unavoidably contain impurities such as salt. In pure ice, nuclear quantum effects (NQEs) play a crucial role by drastically decreasing the VII-X phase transition pressure [3,4]. However, recent experiments question the role of NQEs in LiCl-doped ice [5]. Whether this is specific to $\mathrm{LiCl}$ ice and the mechanism by which the quantum behavior of the protons can be hindered is still an issue.

At high pressure, the two molecular phases of ice, proton disordered ice VII and proton ordered ice VIII [6,7], transform to ice $\mathrm{X}$, the only known atomic phase of ice. Below the transition, the oxygens form a body-centered-cubic structure, and the hydrogen bonds are characterized by a prototypical double-well proton transfer potential. As the atoms get closer under the effect of increasing pressure, the intrinsic quantum nature of the protons produces more evident effects and favors the onset of quantum tunneling. Upon further reducing the distance between oxygens, the proton potential degenerates into a single-well potential [8], giving rise to a symmetric hydrogen bond, where the hydrogen is located midway between two neighboring $\mathrm{O}$ atoms. By including NQEs, the transition pressure $P_{t}$ is drastically reduced from about $90 \mathrm{GPa}$ as classically predicted [9] to approximately $60 \mathrm{GPa}$ [10-16], that is, the pressure for which the zero-point energy equals the barrier height $[3,4]$. However, the effect of a perturbation on a prototype of structural quantum effects in crystals is not $a$ priori trivial from a fundamental point of view.

Recent studies [5] on LiCl-water solutions at different salt to water ratios pointed out that the properties of ice change drastically when $\mathrm{LiCl}$ salt is homogeneously included into ice VII and that the transition pressure to the phase $\mathrm{X}$ strongly depends on the presence of ionic impurities. Here, we report high resolution Raman scattering experiments on $\mathrm{NaCl}$ ice up to Mbar pressures. These challenging conditions have been achieved by the use of a diamond anvil cell equipped with extra low-fluorescence synthetic diamonds and by fast quenching of a $5 \mu \mathrm{m}$ droplet of $2 \% \mathrm{NaCl}$ water solution in order to produce ice VII-doped ice following the procedure described in the Supplemental Material $[5,17,18]$. The upper panel of Fig. 1 shows the $200-1000 \mathrm{~cm}^{-1}$ region of the Raman spectra recorded at different pressures up to $130 \mathrm{GPa}$. The oxygen-oxygen $T_{2 g}$ vibrational mode, which is indicative of the cupritelike structure of phase $X$, clearly appears at $87 \pm 5 \mathrm{GPa}$, similarly to what is observed in $\mathrm{LiCl}$ ice [5]. Thus, the presence of small quantities of salt impurities (here, $1 \mathrm{NaCl}$ for $53 \mathrm{H}_{2} \mathrm{O}$ ), which is likely in natural ices, shifts $P_{t}$ by about $30 \mathrm{GPa}$, roughly the same pressure shift as observed when quantum effects are neglected in pure ice $[3,4]$. This observation confirms that the measured shift of $P_{t}$ in $\mathrm{LiCl}$ ice is actually a general effect of ionic impurities on the ice lattice.

We address this issue by comparing simulations that do or do not include NQEs and quantify their impact on the transition. The first kinds of calculations are done via quantum thermal bath $a b$ initio molecular dynamics (QTB-AIMD) [19,20]; this semiclassical approximation quantitatively describes the VII-X transition in pure ice [4]. The second kinds of calculations are standard $a b$ initio molecular dynamics (AIMD). In both cases, the atomic forces are computed within the density functional theory (DFT), via the generalized gradient approximation [21], as implemented in the QUANTUM ESPRESSO package [22]. Simulations including $\mathrm{NaCl}$ or $\mathrm{LiCl}$ impurities were run over a time length of about 25 ps with a 0.484 fs integration time step. Our simulation cell contains 53 water molecules and one $\mathrm{LiCl}$ or $\mathrm{NaCl}$ pair, corresponding to a concentration of $2 \%$ mol. The $\mathrm{Li}^{+}$ion is small enough to occupy an interstitial site within the oxygen lattice, while $\mathrm{Cl}^{-}$is substituted for a water molecule [17]. Two configurations are considered in our calculations for the larger $\mathrm{Na}^{+}$cation: an interstitial site (I), as for $\mathrm{Li}^{+}$, and a substitutional site (S), where $\mathrm{Na}^{+}$ replaces a water molecule, as does $\mathrm{Cl}^{-}$(the method and initial configurations are described in the Supplemental Material).

The incorporation of salt has non-negligible effects on the hydrogen bonds, as shown by the $\mathrm{OH}$ pair correlation function (PCF) (see the figure and related description in the Supplemental Material). In particular, the first peak of the 


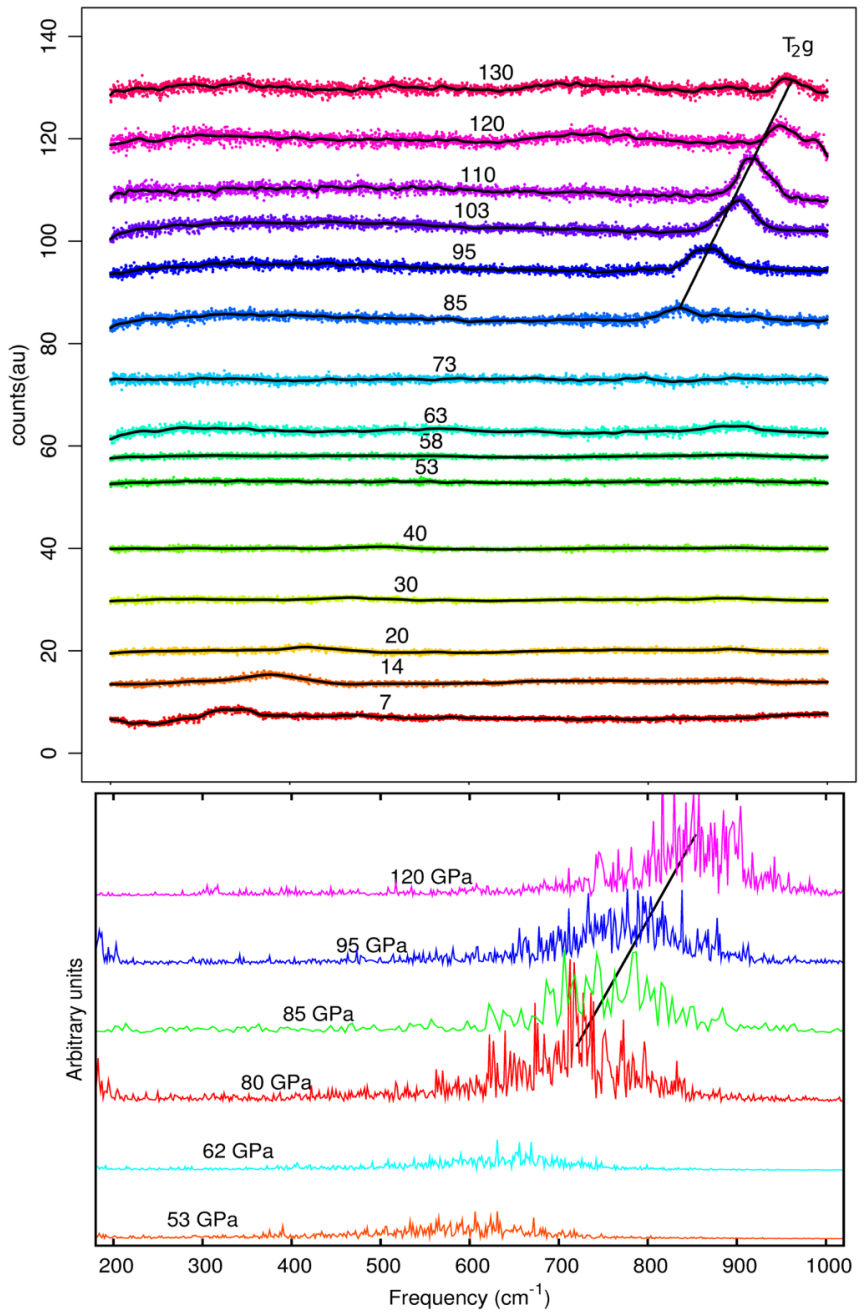

FIG. 1. Top panel: Raman scattering measurements in the $200-1000 \mathrm{~cm}^{-1}$ range as a function of pressure up to $130 \mathrm{GPa}$ for a $2 \%$ molar concentration of $\mathrm{NaCl}$. Lower panel: Calculated $T_{2 g}$ vibrational mode (from QTB-AIMD) in $\mathrm{NaCl}$ ice (S).

PCF is shifted to shorter distances while its second peak, corresponding to the hydrogen bond mean length, is shifted to higher distances with respect to pure ice. With increasing pressure, the two peaks of the PCF broaden significantly and merge into a single one.

Upon increasing pressure, the $\mathrm{OH}$ stretching mode softens, going through a minimum, which determines $P_{t}$ [4,9,12-15]. The pressure evolution of this vibrational mode, calculated from the molecular dynamics trajectories, is shown in Fig. 2 for pure and salty ices (with either $\mathrm{LiCl}$ or $\mathrm{NaCl}$ ), along with experimental results. In parallel, the computed frequency of the $T_{2 g}$ mode in $\mathrm{NaCl}$-ice $\mathrm{X}$ increases with pressure, in agreement with Raman measurements (Fig. 1, lower panel).

In pure ice, the transition pressure $P_{t}$ as predicted by AIMD is about $30 \mathrm{GPa}$ higher than the one yielded by QTB-AIMD (around $60 \mathrm{GPa}$ ), the latter one being instead in very good agreement with experiments. In salty ices, according to QTBAIMD, the VII to X transition occurs at approximately $90 \mathrm{GPa}$ for both $\mathrm{LiCl}$ and $\mathrm{NaCl}$ ices, again in very good agreement with experiments [5]. Moreover, $P_{t}$ is also independent of the actual position of $\mathrm{Na}^{+}$, whether interstitial or substitutional. Hence,

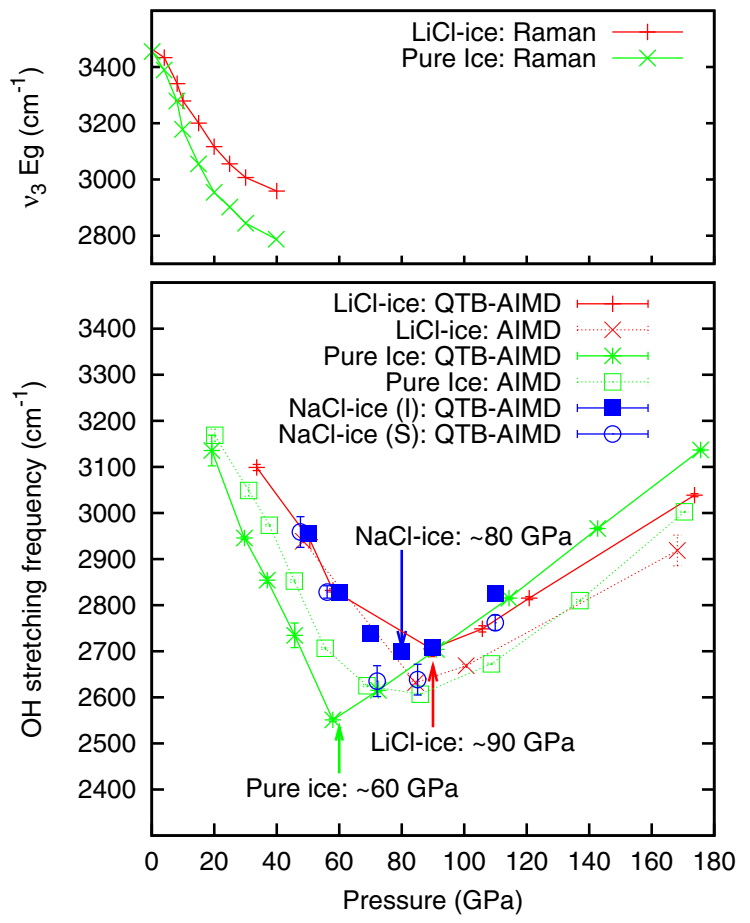

FIG. 2. Top panel: Raman measurements of the $\mathrm{OH}$ stretching mode $\left(v_{3} E_{g}\right)$ in pure and $\mathrm{LiCl}$ ices (8\% mol concentration [5]). Lower panel: $\mathrm{OH}$ stretching mode $\left(\mathrm{in} \mathrm{cm}^{-1}\right.$ ) as a function of pressure (in GPa) obtained from QTB-AIMD (solid lines) and AIMD (dotted lines), in pure ice (green) [4], in $\mathrm{LiCl}$ ices (red), and in $\mathrm{NaCl}$ ices [blue dots: solid squares for interstitial $\mathrm{Na}$ (I), open circles for substitutional $\mathrm{Na}(\mathrm{S})]$. The transition pressure is indicated by the minimum of the curve.

the similarities between $\mathrm{LiCl}$ and $\mathrm{NaCl}$ ices indicate that the shift of $P_{t}$ does not depend, within our statistical uncertainty, on the type or size of the ionic impurity introduced in ice. Even more unexpectedly, we observe that in salty ices, AIMD gives similar results to QTB-AIMD, in particular, yielding $P_{t} \simeq 90 \mathrm{GPa}$, which raises the fundamental question of the resilience of NQEs in those systems.

A natural explanation of this puzzling phenomenon, as given in Ref. [5] for $\mathrm{LiCl}$ ice, is that the local impurity causes a distortion of the neighboring ice structure, and perturbs the motion of hydrogen atoms nearby, which prevents the symmetrization of all other hydrogen bonds in the system. Indeed, the presence of the cation affects the structure of the neighboring oxygen lattice: The oxygen-oxygen distances are slightly larger than in the rest of the system (about 2\%-4\%; see Fig. 3). The angles of the oxygen lattice are also modified in the vicinity of the cation (see the Supplemental Material). In any case, the geometric perturbation is short ranged, independently of the cation ( $\mathrm{Li}$ or $\mathrm{Na}$ ) and its specific location. In particular, the mean $\mathrm{O}-\mathrm{O}$ distance, which has been recognized as a critical parameter [8], is equal in salty and pure ices at the same pressure for the overwhelming majority of $\mathrm{O}$ atoms, apart from the $\simeq 6 \%$ fraction of $\mathrm{H}$ atoms close enough to be impacted by salt inclusion. Accordingly, the ion-induced local distortion cannot explain why the transition is shifted to much larger pressures. 


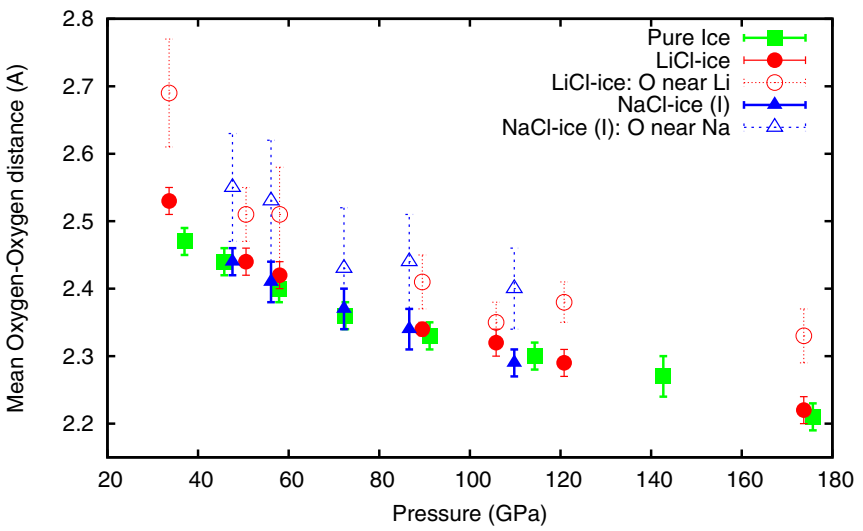

FIG. 3. Mean oxygen-oxygen distance (corresponding to the O$\mathrm{H} \cdots \mathrm{O}$ bond) in pure ices (squares), in $\mathrm{LiCl}$ ices (solid circles), and in $\mathrm{NaCl}$ ices (solid triangles). Open circles and triangles correspond to the mean $\mathrm{O}-\mathrm{O}$ distances near the cation.

The analysis of the probability distribution $P(\xi)$, where $\xi$ is the difference between the covalent $\mathrm{OH}$ and the hydrogen $\mathrm{H} \cdots \mathrm{O}$ bond lengths and is a good order parameter for the transition [23-25], shows that in phase VII, below the transition, the probability distribution averaged over all protons in all salty ices has the same shape (Fig. 4) and is similar to that in pure ice at higher pressure. Hence, the symmetrization of hydrogen bonds is impacted, irrespective of the type and sites of the ionic impurities, further confirming that the salt-induced upshift of $P_{t}$ cannot be purely ascribed to local geometric distortions.

Finally, the overall trend of the proton dynamics is little affected by the salt incorporation. In ice VII, the protons move incoherently from one another, consistent with the disordered character of this phase, while a more coherent motion is recovered in the crystalline phase $\mathrm{X}$ (velocityvelocity correlation functions are shown in the Supplemental Material). It is therefore unlikely that a local perturbation could shift by about $30 \mathrm{GPa}$ the onset of a coherent motion for all protons in the system. In conclusion, structural and dynamical data conspire to exclude that local steric effects could explain the major and largely cation-independent ( $\mathrm{Li}$ or $\mathrm{Na}$ ) upshift of $P_{t}$.

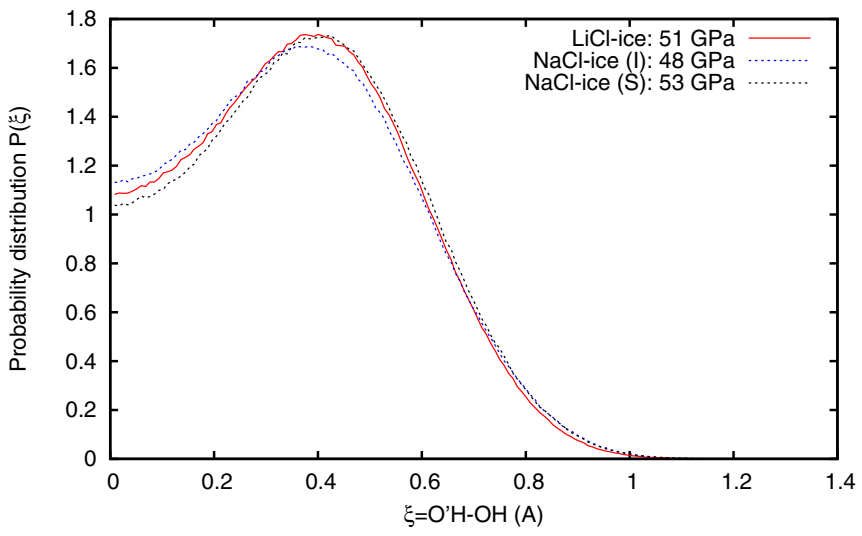

FIG. 4. Probability distribution $P(\xi)$ where $\xi$ is the length difference between the covalent $\mathrm{OH}$ and the hydrogen $\mathrm{H} \cdots \mathrm{O}$ bonds, in $\mathrm{LiCl}$ and $\mathrm{NaCl}$ ices at approximately $50 \mathrm{GPa}$.
We therefore focus on the ion-induced electric field [26] as a long-ranged quantity that could potentially suppress the quantum behavior of protons in ice. In order to check the order of magnitude of this electric field, we compute the electric potential, macroscopically averaged in pure and salty ice. In pure ice, the mean electric field is null, while in salty ice a nonzero mean electric field becomes apparent in the whole sample above the statistical noise, with an intensity varying between 0.2 and $1 \mathrm{~V} / \AA$ (see the Supplemental Material).

In order to test whether a long-range electric perturbation is actually the key to the disappearance of NQEs in ice and the consequent upshift of $P_{t}$, we study the general and simpler case of pure ice under a uniform external electric field. The effect of such a field on proton conduction has already been studied in ices Ih and XI [27]. Here, we focus on its consequences on proton tunneling at much higher pressures, which are by far less known, by performing total energy calculations through the modern polarization theory [28,29]. Then, we derive the mean effective potential felt by a proton along the hydrogen bond double well, for different values of the electric field, which we compare to the same quantity computed for both salty ices. A uniform field of about $0.05 \mathrm{~V} / \AA$ fits the doublewell asymmetry found for salty ices at $2 \%$ concentrations. The results displayed in Fig. 5 show that in pure ice with no electric field, the mean potential is symmetric, with a barrier height of approximately $0.45 \mathrm{eV}$ at low pressure $(10 \mathrm{GPa})$. In contrast, in both salty ices and pure ice under an electric field, one well is lower than the other [30]. Apart from the asymmetry, other differences are mainly in the repulsive part of the potential, which is not essential to the tunneling properties of the protons.

$\mathrm{LiCl}$ ice, $\mathrm{NaCl}$ ices, and pure ice under a uniform electric field share the asymmetry of the proton potential. A general description encompassing the previous physical systems is given by a simple one-dimensional model [23-25,31] of a quantum particle in a double well in the presence of a homogeneous electric field $E$, and focuses on the effect of the asymmetry induced by $E$ [32]. It reads

$$
V(x)=a x^{4}+b\left(P-P_{0}\right) x^{2}+V_{0}+E x,
$$

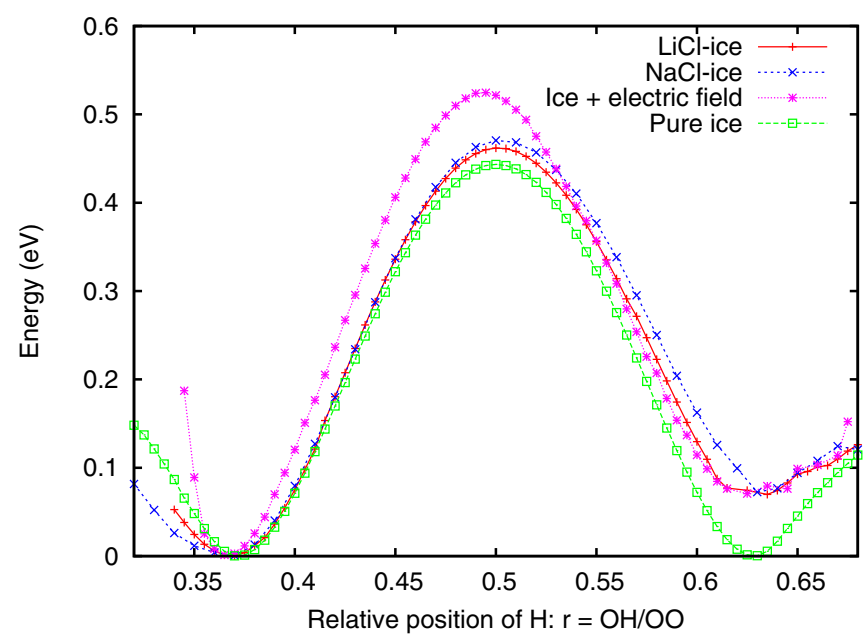

FIG. 5. Potential barrier obtained for the protons in $\mathrm{LiCl}$ ices (red) and in $\mathrm{NaCl}$ ices (blue), in ice under an external uniform electric field, $E=0.05 \mathrm{~V} / \AA$ A (purple), and in pure ice (green) at approximately $10 \mathrm{GPa}$. 


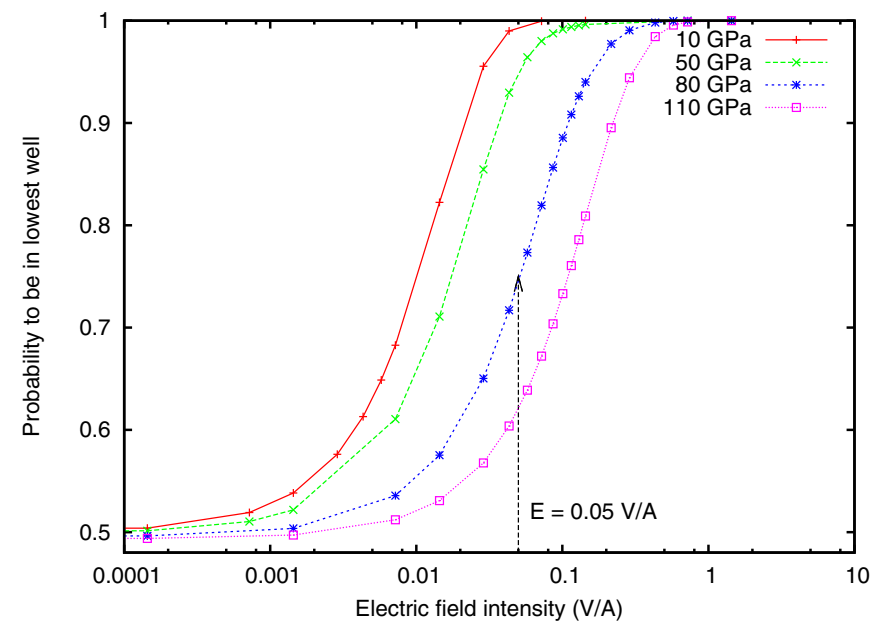

FIG. 6. Probability for the proton to be in the lowest well of the potential in Eq. (1), vs electric field $E$, for different pressures.

where $x$ is the particle position along the $\mathrm{O}-\mathrm{O}$ direction, $P$ the pressure, and $a, b, P_{0}$, and $V_{0}$ are fitting parameters adjusted to the mean potential energy calculated in pure ice [4]. This leads to a textbook quantum mechanics problem [see the Supplemental Material for details about the one-dimensional (1D) model]. The numerical solutions of the corresponding time-independent Schrödinger equation, for varying $P$, show that the probability for the particle to be in the lowest well evolves rapidly with increasing electric field from $0.5-$ corresponding to the quantum tunneling regime-to 1 , where the proton localizes in one well (Fig. 6). Within this general model, even a small electric field drastically reduces the proton tunneling rate between the two potential wells [31], making the most probable position of protons indistinguishable from the equilibrium position of their classical counterparts. For instance, a homogeneous electric field of $0.05 \mathrm{~V} / \AA$ in pure ice implies that the probability of finding the particle in one well is about $75 \%$ at $80 \mathrm{GPa}$ (Fig. 6). As the electric field increases the particle localization, the symmetrization of the hydrogen bond can only be attained by increasing the pressure, in agreement with the experimental findings.

In conclusion, our study provides a fully consistent experimental and theoretical description of the suppression of the quantum behavior of protons in salty ices. The incorporation of salt in high-pressure ice generates a long-range electric field that affects all the protons in the system, beyond the local distortion. This electric field modifies the potential landscape around the protons, making it asymmetric instead of symmetric as in pure ice. Even a small asymmetry is able to localize the hydrogen nuclei and to largely suppress tunneling along the $\mathrm{O}-\mathrm{O}$ direction, shifting the transition to ice $\mathrm{X}$ to higher pressures. This explains why the disappearance of nuclear quantum effects on protons occurs at rather low salt concentrations. A simple textbook model captures the essence of the problem and demonstrates that NQEs are strongly affected by the asymmetry inherent to the electric field. This allows us to infer a general trend on the effect of dilute impurities on realistic planetary ices, where the transition pressure to ice $\mathrm{X}$ can be significantly displaced by salt concentrations as small as $1 \%$.

This work was granted access to the high-performance computing resources of CINES under the allocation 2015096719 made by GENCI. Y.B. acknowledges financial support from the Conseil Régional d'Ile-de-France (France) through the DIM Oxymore.
[1] J. D. Anderson, G. Schubert, R. A. Jacobson, E. L. Lau, W. B. Moore, and W. L. Sjogren, Science 281, 2019 (1998); K. K. Khurana, M. G. Kivelson, D. J. Stevenson, G. Schubert, C. T. Russell, R. J. Walker, and C. Polanskey, Nature (London) 395, 777 (1998); N. Schilling, K. K. Khurana, and M. G. Kivelson, J. Geophys. Res. 109, E05006 (2004); B. G. Bills, Icarus 175, 233 (2005).

[2] J. J. Lissauer, Nature (London) 419, 355 (2002).

[3] M. Benoit, D. Marx, and M. Parrinello, Nature (London) 392, 258 (1998).

[4] Y. Bronstein, P. Depondt, F. Finocchi, and A. M. Saitta, Phys. Rev. B 89, 214101 (2014).

[5] L. E. Bove, R. Gaal, Z. Raza, A. A. Ludl, S. Klotz, A. M. Saitta, A. F. Goncharov, and P. Gillet, Proc. Natl. Acad. Sci. USA 112, 8216 (2015).

[6] W. F. Kuhs, J. L. Finney, C. Vettier, and D. V. Bliss, J. Chem. Phys. 81, 3612 (1984).

[7] R. J. Hemley, Annu. Rev. Phys. Chem. 51, 763 (2000).

[8] M. Benoit and D. Marx, Solid State Ionics 125, 23 (1999); ChemPhysChem 6, 1738 (2005).

[9] M. Bernasconi, P. L. Silvestrelli, and M. Parrinello, Phys. Rev. Lett. 81, 1235 (1998).
[10] W. B. Holzapfel, J. Chem. Phys. 56, 712 (1972).

[11] P. Pruzan, J. Mol. Struct. 322, 279 (1994).

[12] K. Aoki, H. Yamawaki, and M. Sakashita, Phys. Rev. Lett. 76, 784 (1996).

[13] A. F. Goncharov, V. V. Struzhkin, M. S. Somayazulu, R. J. Hemley, and H. K. Mao, Science 273, 218 (1996); A. F. Goncharov, V. V. Struzhkin, H. K. Mao, and R. J. Hemley, Phys. Rev. Lett. 83, 1998 (1999).

[14] V. V. Struzhkin, A. F. Goncharov, R. J. Hemley, and H. K. Mao, Phys. Rev. Lett. 78, 4446 (1997).

[15] M. Song, H. Yamawaki, H. Fujihisa, M. Sakashita, and K. Aoki, Phys. Rev. B 60, 12644 (1999).

[16] P. Loubeyre, R. LeToullec, E. Wolanin, M. Hanfland, and D. Hausermann, Nature (London) 397, 503 (1999).

[17] S. Klotz, L. E. Bove, T. Straessle, T. C. Hansen, and A. M. Saitta, Nat. Mater. 8, 405 (2009).

[18] See Supplemental Material at http://link.aps.org/supplemental/ 10.1103/PhysRevB.93.024104 for detailed information about: (i) the experimental procedure and the theoretical method; (ii) the structure of the simulated systems; (iii) the coherent and incoherent vibrational spectra; (iv) the one-dimensional model.

[19] M. Ceriotti, G. Bussi, and M. Parrinello, Phys. Rev. Lett. 103, 030603 (2009). 
[20] H. Dammak, Y. Chalopin, M. Laroche, M. Hayoun, and J. J. Greffet, Phys. Rev. Lett. 103, 190601 (2009).

[21] J. P. Perdew, K. Burke, and M. Ernzerhof, Phys. Rev. Lett. 77, 3865 (1996).

[22] P. Giannozzi et al., J. Phys.: Condens. Matter 21, 395502 (2009).

[23] E. Kryachko, M. Eckert, and G. Zundel, J. Mol. Struct. 270, 33 (1992).

[24] J. A. Morrone, L. Lin, and R. Car, J. Chem. Phys. 130, 204511 (2009).

[25] L. Lin, J. A. Morrone, and R. Car, J. Stat. Phys. 145, 365 (2011).

[26] J. Klimes, D. R. Bowler, and A. Michaelides, J. Chem. Phys. 139, 234702 (2013).
[27] G. Cassone, P. V. Giaquinta, F. Saija, and A. M. Saitta, J. Phys. Chem. B 118, 4419 (2014); 118, 12717 (2014).

[28] M. V. Berry, Proc. R. Soc. London, Ser. A 392, 45 (1984).

[29] P. Umari and A. Pasquarello, Phys. Rev. Lett. 89, 157602 (2002).

[30] The potential was computed for $\mathrm{LiCl}$ and $\mathrm{NaCl}$ ices (I and $\mathrm{S}$ ). These systems have very similar potentials, as can be seen in Fig. 5, confirming the general effect of salt impurities.

[31] T. Maslowski, A. Drzewinski, J. Ulner, J. Wojtkiewicz, M. Zdanowska-Fraczek, K. Nordlund, and A. Kuronen, Phys. Rev. E 90, 012135 (2014).

[32] P. G. Johannsen, J. Phys.: Condens. Matter 10, 2241 (1998). 